\title{
Control por Modos Deslizantes de la Relación de Exceso de Oxígeno en una PEMFC Interconectada a un Motor de CD mediante un Convertidor Boost
}

\section{Sliding Mode Control of Oxygen Excess Ratio on a PEMFC Interconnected to a DC Motor Using a Boost Converter}

\author{
CERVERA-CEBALlos, María†*, RODRÍGUEZ-BLANCO, Marco A., VAZQUEZ-ÁVILA, José L. \\ y ALAZKI, Hussain
}

Universidad Autónoma del Carmen, Facultad de Ingeniería y Tecnologia

ID $1^{\text {er }}$ Autor: María, Cervera-Ceballos / ORC ID: 0000-0002-5830-0407, Researcher ID Thomson: P-7234-2019, CVU CONACYT ID: 166309

ID $1^{\text {er }}$ Coautor: María, Cervera-Ceballos / ORC ID: 0000-0003-3641-6895, Researcher ID Thomson: U-6476-2017, CVU CONACYT: 51908

ID $2^{\text {do }}$ Coautor: José L., Vazquez-Ávila / ORC ID: 0000-0002-9654-2431, Researcher ID Thomson: P-7994-2019; CVU CONACYT ID: 102415

ID $3^{\text {er }}$ Coautor: Hussain, Alazki / ORC ID: 0000-0002-1960-3624, Researcher ID Thomson: M-1706-2017

DOI: $10.35429 / J E A .2019 .19 .6 .25 .32$

Recibido: 19 de Abril 2019; Aceptado 30 de Junio, 2019

\section{Resumen}

En este trabajo se utiliza una celda de combustible de membrana de intercambio de protones (PEMFC) para suministrar de manera sustentable la energía a un motor de CD de imán permanente y para garantizar la eficiencia del funcionamiento en la PEMFC se manipula el flujo de oxígeno en la entrada del cátodo. Se cuenta con un lazo de control lineal PI en un convertidor CD/CD tipo Boost conectado exclusivamente a la PEMFC para regular la velocidad de un motor de $\mathrm{CD}$ en presencia de disturbios en la carga. Adicionalmente, se diseña un control no lineal por Modos Deslizantes (SMC) regulando la relación de exceso de oxígeno $\lambda \mathrm{O}_{2}$ para mantenerse dentro de la región óhmica de la curva de polarización, evitar la falta de oxígeno en el cátodo ante cambios en la potencia exigida por el motor y prevenir daños en los componentes de la PEMFC. Los resultados son validados utilizando los modelos internos de la PEMFC y la electrónica de potencia de Matlab/Simulink.

Celdas de combustible, Control del suministro de oxígeno, Control por modos deslizantes

\begin{abstract}
In this work, a proton exchange membrane fuel cell (PEMFC) is used to electric energy supply to a permanent magnet DC motor in a sustainable way and the inlet air flow in the cathode is manipulated to ensure the PEM fuel cells efficient operation. A boost-type DC/DC converter is connected to the PEMFC, it is used with a PI linear control loop to regulate the speed of the DC motor under some possible load disturbances. In addition, a nonlinear sliding mode control (SMC) is designed for regulated the excess oxygen ratio considering constant the temperature and humidity of membrane, to achieve the PEM full cell operate in the ohmic region of the polarization curve, to avoid the oxygen starvation at the cathode and to prevent damage to fuel cell components. The results are validated using the internal models of the PEMFC and the power electronics from SimPowerSystems library of Matlab/Simulink.
\end{abstract}

Fuel cell, Oxygen supply control, Sliding Mode Control

Citación: CERVERA-CEBALLOS, María, RODRÍGUEZ-BLANCO, Marco A., VAZQUEZ-ÁVILA, José L. y ALAZKI, Hussain. Control por Modos Deslizantes de la Relación de Exceso de Oxígeno en una PEMFC Interconectada a un Motor de CD mediante un Convertidor Boost. Revista de Aplicaciones de la Ingeniería. 2019. 6-19: 25-32

\footnotetext{
* Correspondencia del Autor (Correo electrónico: mcervera.mc@ gmail.com)

$\dagger$ Investigador contribuyendo como primer autor.
} 


\section{Introducción}

Actualmente el uso de Celdas de Combustible (FC) en aplicaciones portátiles ha incrementado significativamente. Lo anterior se debe a las nuevas tecnologías de generación de hidrogeno y a los nuevos materiales para el diseño de las celdas que permiten un diseño compacto y eficiente (Kunusch et al., 2012; O'hayre et al., 2016; Hirschenhofer et al., 1998).

Existen muchos campos de investigación sobre celdas de combustibles y el tipo de celda va a depender de la aplicación de interés como las celdas de solución óxido de sólido SOFC para aplicaciones de gran potencia, o como las celdas de membrana de polímero conductora de protones PEMFC adecuadas para aplicaciones móviles, dado que funciona a bajas temperaturas, manejo y ensamblaje menos complejos, electrolito no corrosivo, adecuado funcionamiento al aire atmosférico, electrolito sólido y seco, manejo de medianas potencias y buena tolerancia a la diferencia de presión de los reactivos, entre otro.

Aunque el inconveniente son las diversas gestiones para el buen desempeño como tolerancia a las partículas de azufre, humidificación de gases reactivos, temperatura, hidratación del electrolito y suministro de reactivos de oxígeno e hidrógeno. De lo anterior, la gestión más importante para controlar, con relación a la dinámica rápida, es la gestión del suministro de hidrogeno y oxígeno, por lo que en la literatura se ha publicado muchos artículos con respecto a este tópico (Zhan et al., 2008; Glazer et al., 2013; Feroldi et al., 2006). Sin embargo, el control del oxígeno ha sido mayormente abordado debido principalmente a las condiciones menos peligrosas que conllevar a manejar el hidrogeno (Feroldi et al., 2006).

En este sentido, existen muchas referencias orientadas a aplicaciones móviles que emplean mayormente el conjunto celdaconvertidor-motor (Glazer et al., 2013) en donde un motor de $\mathrm{CD}$ es conectado a través de un convertido de $\mathrm{CD} / \mathrm{CD}$ y éste a su vez conectado a una celda, la cual es controlada exclusivamente por el suministro de oxígeno Similar a lo anterior en (Alnaib et al., 2018) se presenta otro trabajo en donde se muestra un esquema similar pero utilizando un motor de CD de excitación separada en donde se alimenta el campo por una batería independiente.
El problema encontrado en las dos referencias arriba es que la factibilidad no es optimizada dado que el control de oxigeno no garantiza el buen desempeño de la celda y por lo tanto la degradación temprana de las membranas será inevitable. Existen trabajos mucho más completos como el predictivo adaptativo con filtro robusto (Zumoffen et al., 2010), Control predictivo de modelo restringido (MPC) (Bordons et al., 2006), Control por lógica difusa (Tekin et al., 2007; Williams et 1., 2008; Alnaib et 1., 2018), Control por Redes neuronales (Hatti et al., 2009). Sin embargo, todos los esquemas anteriores necesitan un alto esfuerzo computacional o gran capacidad de almacenamiento de datos, lo cual repercute en plataformas de desarrollo costosas no factibles para aplicaciones móviles.

En este trabajo se propone controlar la velocidad de un motor de $\mathrm{CD}$ de imán permanente conectado a un convertidor $\mathrm{CD} / \mathrm{CD}$ tipo Boost el cual toma la energía de suministro de una celda de combustible PEMPC controlada por la estrategia de modos deslizantes sin compensación de estados. El objetivo de control es mantener la relación de oxigeno constante, y un rango acotado del voltaje de la celda sin afectar la velocidad del motor. Así mismo, que el control desarrollado tenga un mínimo esfuerzo computacional factible para aplicaciones móviles. El trabajo es validado mediante un análisis teórico y resultados de simulación.

\section{Sistema de celda de combustible}

\section{Estructura propuesta}

En este trabajo se plantea la estructura de control que se muestra en la figura 1, se proponen dos lazos de control considerando variaciones en la carga con el objetivo de mantener en un valor óptimo la relación de exceso de oxígeno $\left(\lambda_{\mathrm{O}_{2}}\right) \mathrm{de}$ una Celda de Combustible de Membrana de Intercambio de Protones (PEMFC) usada como fuente de alimentación de un motor de CD de imán permanente. Se proponen dos lazos de control, el primero corresponde al controlador por modos deslizantes, siendo su principal objetivo mantener en un valor óptimo la relación de exceso de oxígeno de una celda de combustible de membrana de intercambio de protones. El segundo lazo, se trata de un PI, este se encarga de mantener la velocidad deseada en el motor, considerando variaciones de carga. 


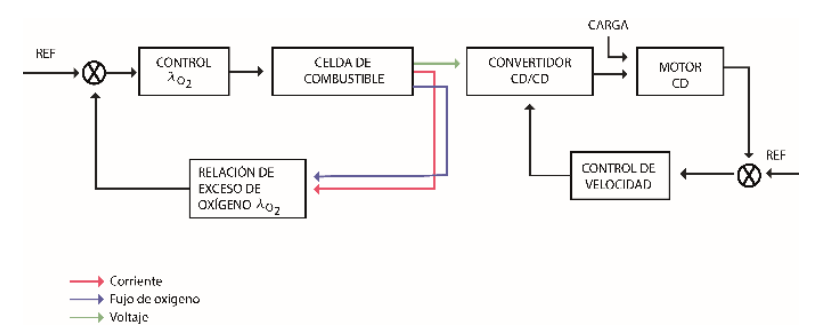

Figura 4 Estructura de control del sistema PEMFC conectado a un motor $\mathrm{CD}$

\section{Celda de combustible}

En una celda de combustible, se genera electricidad mediante la reacción entre el hidrógeno y el oxígeno formando agua. La estructura básica de una Celda de Combustible (FC) está compuesta por dos electrodos, un ánodo por donde se suministra el hidrógeno y un cátodo en donde entra el oxígeno, los cuales se encuentran separados por un electrolito. El hidrógeno puede ser entregado a partir de un tanque de hidrógeno presurizado mientras que el suministro de oxígeno utiliza comúnmente un compresor. La falta de presión de combustible u oxígeno para suministrar las diferentes demandas de carga que pueden requerirse provoca una caída de voltaje. Si existe una escasez de reactivos, los materiales de la celda podrían dañarse (García et al., 2010). En la figura 2.1 se muestra el esquema de una celda de combustible.

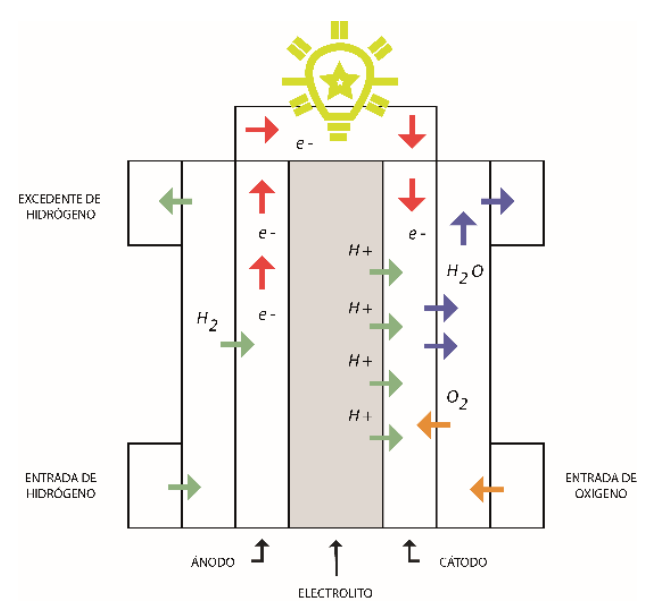

Figura 5 Estructura de una celda de combustible

En el ánodo se oxida el hidrógeno separándose en electrones y protones. Los protones se trasladan al cátodo a través de la membrana, mientras que los electrones fluyen a través de un circuito externo, creando electricidad.

En el cátodo se combinan los electrones procedentes del circuito externo y los protones que pasaron a través de la membrana con el oxígeno produciendo agua.

\section{Convertidor CD/CD}

La celda de combustible proporciona un voltaje muy pequeño, aproximadamente $0.7 \mathrm{~V} /$ celda dependiendo de las condiciones de operación y carga, para obtener mayor voltaje se apilan múltiples celdas en serie formando una pila o stack (en inglés) de celdas de combustible (Larminie et al., 2003). El voltaje total de la pila es el número de celdas multiplicado por el voltaje de celda promedio (Pukrushpan et al., 2004). Por otra parte, se puede utilizar un convertidor DC/DC como acondicionador de energía para elevar el voltaje de la celda de combustible (Somkun et al., 2015).

El convertidor Boost sirve para obtener un voltaje de alimentación continuo de mayor valor que el que ingresa. El diagrama del convertidor Boost se muestra en la figura 2.2, este contiene dos dispositivos semiconductores: el diodo para evitar que la corriente de descarga del capacitor se regrese y un elemento de conmutación rápida, como un IGBT, además se usa un capacitor y un inductor para almacenar energía. El procedimiento para el diseño se puede encontrar en (Simon et al., 2005).

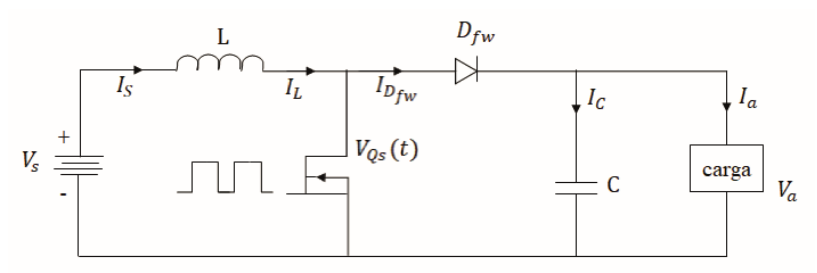

Figura 6 Circuito esquemático del convertidor elevador

\section{Control en el suministro de oxígeno}

El control en el suministro de aire de una celda de combustible PEM ante diferentes cambios en la demandada de carga es importante para garantizar un buen funcionamiento dinámico y prevenir la falta de oxígeno. La cantidad de oxígeno baja cuando la corriente demandada por la carga aumenta, esto causa caídas de voltaje, además puede dañar la membrana y reducir la eficiencia del sistema FC. A este fenómeno se le conoce mayormente como oxygen starvation.

Una manera eficiente de garantizar el nivel adecuado de oxígeno es regulando la relación de exceso de oxígeno $\lambda_{\mathrm{O}_{2}}$ (Glazer et al., 2013; Zumoffen et al., 2010, Pukrushpan et al., 2004), definida como:

$\lambda_{\mathrm{O}_{2}}=\frac{\mathrm{w}_{\mathrm{O}_{2}, \text { in }}}{\mathrm{w}_{\mathrm{O}_{2}, \text { reacted }}}$ 
Donde $\mathrm{W}_{\mathrm{O}_{2} \text {,in }}$ es el flujo de oxígeno ingresado en el cátodo que depende del flujo de aire liberado por el compresor y $\mathrm{W}_{\mathrm{O}_{2} \text {,reacted }}$ es el flujo consumido debido a la reacción electroquímica que está relacionado con la corriente total de pila $I_{s t}$. A partir de las ecuaciones (2) y (3) se realizar el cálculo de $\mathrm{W}_{\mathrm{O}_{2} \text {,in }}$ y $\mathrm{W}_{\mathrm{O}_{2} \text {,reacted considerando un valor }}$ óptimo para $\lambda_{O_{2}}$ igual a 2 (Pukrushpan et al., 2004). El flujo de oxígeno que entra en el cátodo se calcula como:

$\mathrm{W}_{\mathrm{O}_{2} \text {, reacted }}=M_{O_{2}} \frac{n I_{S t}}{4 F}$

Donde $M_{\mathrm{O}_{2}}=32 \times 10^{-3} \mathrm{Kg} / \mathrm{mol}$ es la masa molar de oxígeno, $\mathrm{n}$ es el número de celdas y $F=96,485 \mathrm{C} /$ moles la constante de Faraday. El cálculo del flujo de oxígeno de entrada se obtiene a partir de:

$\mathrm{W}_{\mathrm{O}_{2}, \text { in }}=W_{a, \text { in }} x_{O_{2}, \text { in }}$

Siendo $x_{O_{2} \text {,in }}$ la fracción de masa del oxígeno y $W_{a, i n}$ el flujo másico del aire seco en la entrada del cátodo, determinadas por las ecuaciones (4) y (5).

$x_{O_{2}, \text { in }}=\frac{y_{O_{2}, i n} M_{O_{2}}}{y_{O_{2}, i n} M_{O_{2}}+\left(1-y_{O_{2}, i n}\right) M_{N_{2}}}$

Donde $M_{N_{2}}=28 \times 10^{-3} \mathrm{Kg} / \mathrm{mol}$ es la masa molar de nitrógeno y $y_{O_{2}, \text { in }}=0.21$ es la fracción molar de oxígeno.

$W_{a, i n}=\frac{1}{1+\omega_{c a, i n}}$ definida por:

Donde la relación de humedad $\omega_{c a, \text { in }}$ está

$\omega_{c a, \text { in }}=\frac{M_{v}}{M_{a, i n}} \frac{P_{v, i n}}{P_{a, i n}}$

Donde $M_{v}=18.02 \times 10^{-3} \mathrm{Kg} / \mathrm{mol}$ es la masa molar de vapor, $P_{v \text {,in }}$ la presión del vapor, la presión del aire seco es $P_{a, i n}$ y la masa molar del aire de entrada $M_{a, i n}$ se obtiene de:

$$
\begin{aligned}
& M_{a, \text { in }}=y_{O_{2}, \text { in }} M_{O_{2}}+\left(1-y_{O_{2}, \text { in }}\right) M_{N_{2}} \\
& P_{v, \text { in }}=\Phi_{\text {in }} P_{\text {sat }}\left(T_{i n}\right) \\
& P_{a, \text { in }}=P_{\text {in }}-P_{v, \text { in }}
\end{aligned}
$$

$P_{\text {sat }}\left(T_{\text {in }}\right)$ representa la presión de saturación del vapor con respecto a la temperatura de entrada $T_{\text {in }}=298.15 \mathrm{~K}$, dicha presión puede ser obtenida de las tablas de saturación del vapor presentadas en (Sonntag et al., 1998) y $\Phi_{\text {in }}$ denota la humedad relativa del aire en la entrada considerada igual a 1 . La presión en la entrada del cátodo $P_{\text {in }}$ se obtiene de [14]:

$P_{\text {in }}=1.0033+2.1 \times 10^{-4} W_{a, \text { in }}-475.7 \times 10^{-6} I_{s t}$

\section{Control por modos deslizantes}

El Controlador de Modo Deslizante (SMC) es un control robusto basado en principios de los Sistemas de Estructura Variable (VSS) que consiste en un conjunto de subsistemas continuos con una lógica de conmutación (Utkin, 1977). En los VSS, el control puede cambiar su estructura, es decir, cambiar en cualquier momento de uno a otro miembro de un conjunto de posibles funciones continuas del estado. El problema del diseño de la estructura variable es seleccionar los parámetros de cada una de las estructuras y definir la lógica de conmutación, las cuales a una a frecuencia muy elevada, idealmente infinita, obliga a las trayectorias del sistema a evolucionar sobre una superficie en el espacio de estado conocida como superficie de deslizamiento.

A este modo de funcionamiento, se lo denomina operación por modos deslizantes y se caracteriza por ser altamente robusto e insensible a determinadas perturbaciones externas e incertidumbres del modelo. Para que exista un modo deslizante en una superficie de discontinuidad entre dos estructuras, las trayectorias a ambos lados de la superficie deben dirigirse hacia la región de deslizamiento $S(t)$ como se muestra en la figura 4.

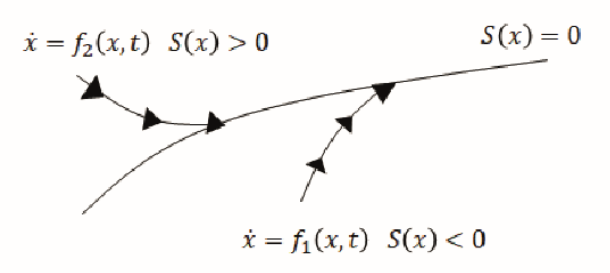

Figura 7 Trayectorias en el plano de fase de un sistema de estructura variable 
En sistemas reales, la frecuencia de conmutación no es infinita, estas conmutaciones generan oscilaciones de alta frecuencia, conocidas como "chattering" en las trayectorias al ir evolucionando sobre la superficie de deslizamiento (Utkin et al., 1996). Sin embargo, este problema puede ser reducido mediante la aplicación de otras técnicas de control.

En este trabajo se diseña una ley de control basada en el Controlador de Modo Deslizante (SMC) cuya tarea es controlar la relación de exceso de oxígeno $\lambda_{\mathrm{O}_{2}}$, que es la salida principal del proceso. La variable manipulada es el flujo de aire requerido y como perturbación del proceso es la demanda de carga que la celda de combustible debe satisfacer al inversor y éste último a la carga del motor de CD. Primero, se define la función de superficie deslizante $(\sigma)$ como:

$\sigma=c e, c>0$

donde $e$ es el error de seguimiento entre la referencia $\left(\lambda_{O_{2}-r e f}\right)$ y la salida $\left(\lambda_{O_{2}}\right)$. El objetivo del control es asegurar que la variable controlada sea dirigida a su valor de referencia.

Posteriormente, se se diseña la ley de control que impulsa la variable controlada a su valor de referencia. La formulación de la ley de control basada en SMC se define como:

$u=K \operatorname{sign}(\sigma)+\bar{u}, K>0$

en donde

$\bar{u}:=\frac{1}{K_{C}} \tau e+\lambda_{O_{2}}$

Donde $\bar{u}$ es la compensación para el sistema, $\tau=1 \mathrm{~s}$ es el tiempo de respuesta de la planta, $K_{c}=1.31$ es la ganancia de compensación y sign es la función signo, definida como:

$$
\operatorname{sign}(\sigma)=\left\{\begin{array}{c}
1 \text { si } \sigma>0 \\
-1 \text { si } \sigma<0
\end{array}\right.
$$

Existe un estudio que prueba que este tipo de control se encuentra estable en la fase de convergencia para la superficie deslizante, en donde se elige una función de Lyapunov donde $V=\frac{1}{2} \sigma^{2}$ y se prueba que cuando $\dot{V}=\sigma \dot{\sigma}<0$, garantizando la estabilidad (Shtessel et al., 2014).

\section{Resultados}

Para el proceso de simulación se considera el modelo detallado de la celda de combustible tipo PEM de $1.2 \mathrm{~kW}$ disponible en SymPowerSystems de Simulink de Matlab así como los componentes electrónicos y el motor de imán permanente que se encuentra en dicha librería. Los datos del motor se muestran la tabla 1.

\begin{tabular}{|l|r|}
\hline \multicolumn{1}{|c|}{ Parámetro } & \multicolumn{1}{c|}{ Valor } \\
\hline Voltaje & $48 \mathrm{~V}$ \\
\hline Corriente & $1.4 \mathrm{~A}$ \\
\hline Torque & $0.11 \mathrm{~N}$ \\
\hline Velocidad nominal & $314 \mathrm{rad} / \mathrm{s}$ \\
\hline
\end{tabular}

Tabla 5 Parámetros del motor DC

Para proporcionar el voltaje de funcionamiento requerido por el motor, se diseña un convertidor CD/CD tipo Boost para elevar el voltaje proporcionado por la celda de combustible de $24 \mathrm{~V}$ a $48 \mathrm{~V}$. En la tabla 2 se encuentran los valores para el convertidor.

\begin{tabular}{|c|c|}
\hline Parámetro & Valor \\
\hline Frecuencia de conmutación & $1 \mathrm{kHz}$ \\
\hline Inductancia & $1613 \mathrm{mH}$ \\
\hline Capacitor & $4000 \mathrm{mF}$ \\
\hline
\end{tabular}

Tabla 6 Parámetros del convertidor CD/CD

En la figura 5, se muestra el sistema completo de control propuesto para el sistema Celda-Convertidor-Motor simulado en Matlab.

La aportación más importante del sistema Celda-Convertidor-Motor propuesto es en especial el SMC aplicado al sistema CeldaConvertidor dado la no linealidad que este sistema involucra tanto por sus las incertidumbres de sus reacciones químicas así como también por los disturbios de carga no lineales que el convertidor involucra, razón por la cual un control robusto ante variaciones de carga e incertidumbres de modelo resulta ser necesario. 


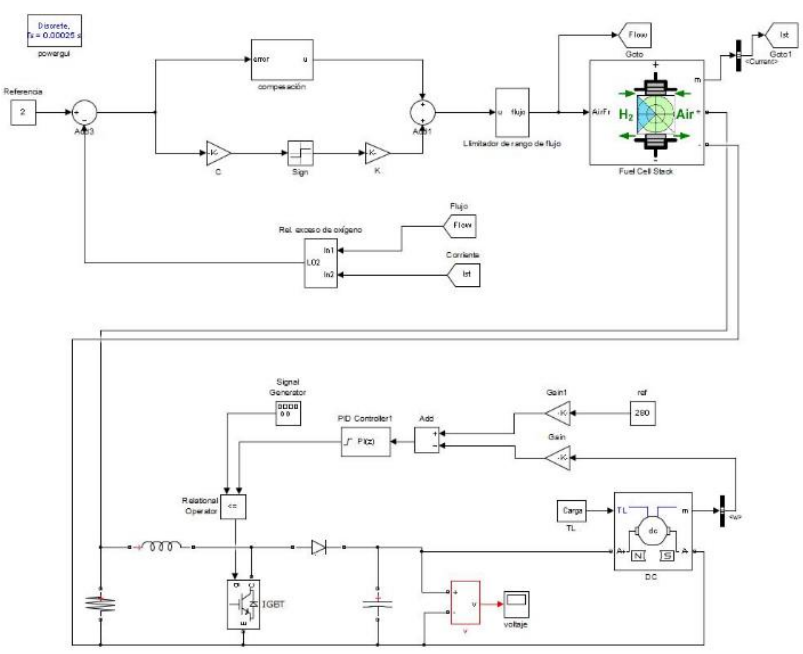

Figura 8 Diagrama a bloques del sistema CeldaConvertido-Motor propuesto

\section{Resultados de simulación}

El motor funciona con un par de carga igual a $0.11 \mathrm{~N}$. m, se consideran variaciones de carga. La figura 6. muestra las señales de velocidad del motor y voltaje de la celda del sistema Convertidor-Motor utilizando un controlador PI cuando una carga de 0.04 es aplicada al transcurrir 5 segundos y otra de 0.08 a los 10 segundos.

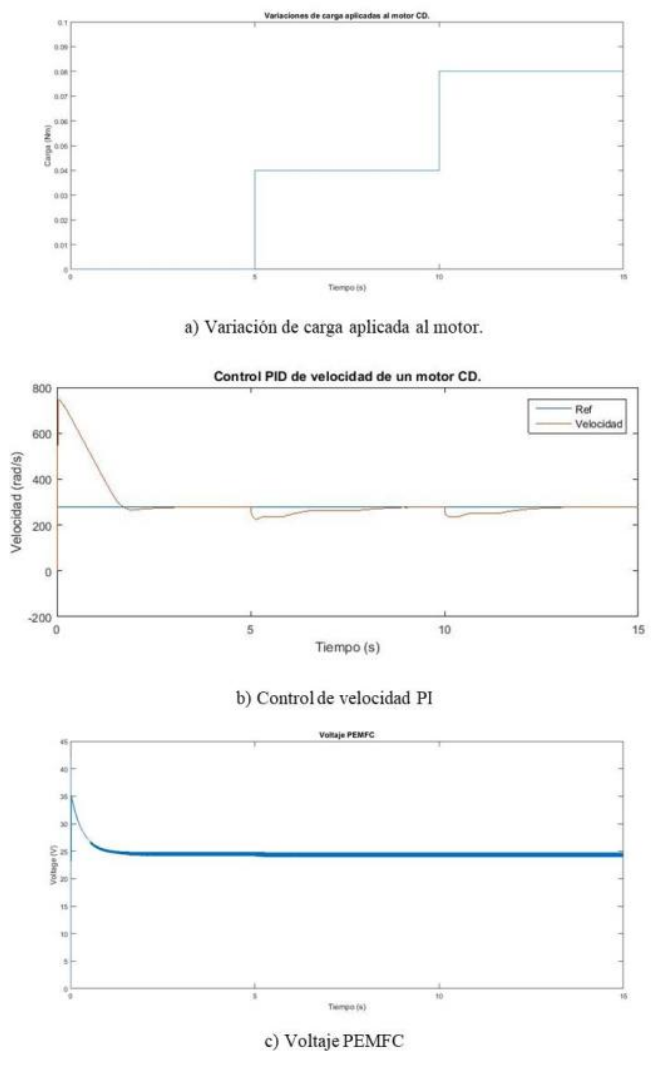

Figura 6 Respuesta del sistema Convertidor-Motor con control PI; a) Variación de carga aplicada al motor, b) Control de velocidad, c) Voltaje a la salida de la PEMFC
Las variaciones de carga en el motor son consideradas como efectos de carga en la celda de combustible. En la figura 7 se muestra la relación de oxígeno $\lambda_{\mathrm{O}_{2}}$ controlada por SMC, considerando estas variaciones de carga, el efecto del chattering, como se mencionó en la sección 3.1 puede ser minimizado por otras técnicas como Super Twisting.

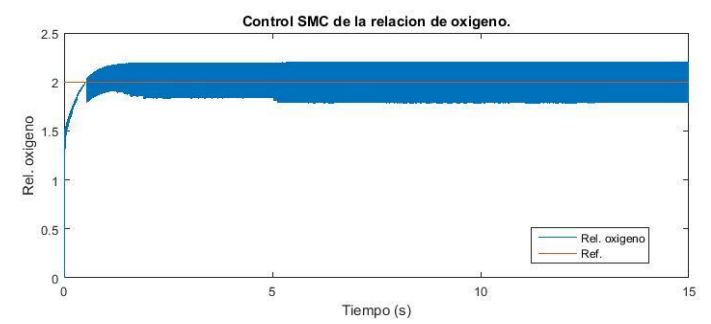

Figura 7 Respuesta del sistema Celda-Convertidor-Motor con control

En la figura 8 se la señal de error de $\lambda_{O_{2}}$, la corriente de la Celda $I_{s t}$.ubicada a un valor intermedio de $25 \mathrm{~A}$ y el flujo de oxígeno con un rango acotado y óptimo de 83 y $93 \mathrm{lpm}$ lo que permite que el sistema pueda funcionar eficientemente. Es importante resaltar al existir un aumento en la corriente de la celda de combustible $I_{s t}$, el flujo de oxígeno de entrada no decae, sino que se mantiene en un valor máximo, lo que evita que haya una falta de oxigenación y por lo tanto, daños en la celda de combustible.

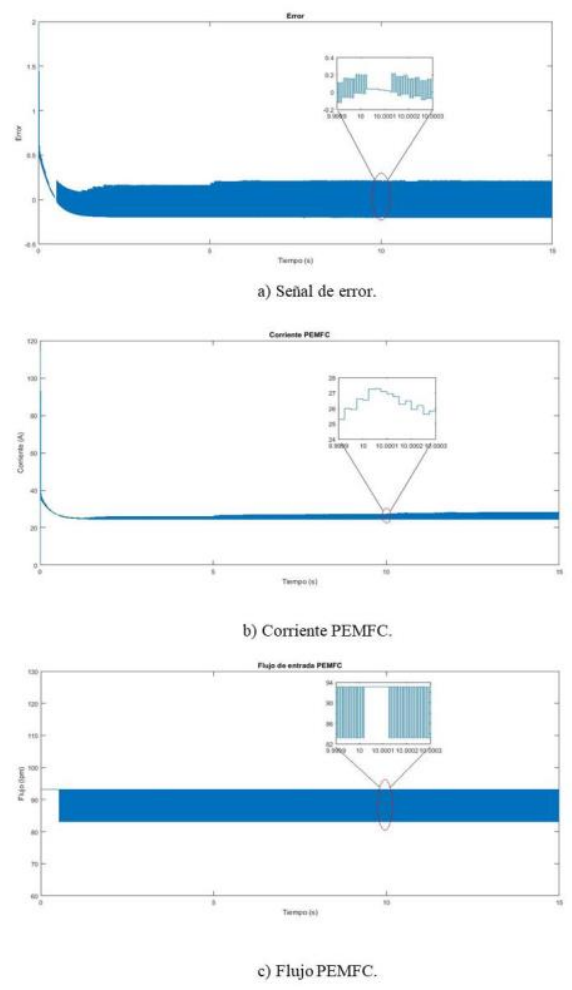

Figura 8 Respuesta del sistema Celda-Convertidor con control SMC; a) señal de error de $\lambda_{\mathrm{O}_{2}}$, b) Corriente Ist de la PEMFC, c) Flujo de oxígeno de entrada a la PEMFC

CERVERA-CEBALLOS, María, RODRÍGUEZ-BLANCO, Marco A. VAZQUEZ-ÁVILA, José L. y ALAZKI, Hussain. Control por Modos Deslizantes de la Relación de Exceso de Oxígeno en una PEMFC Interconectada a un Motor de CD mediante un Convertidor Boost. Revista de Aplicaciones de la Ingeniería. 2019 
Es interesante observar en la figura 7 que, aunque la variable medida de $\lambda_{O_{2}}$ presenta un cierto desvío a frecuencias altas, la señal de se ajusta al valor deseado ante variaciones de carga, lo que guarda un compromiso entre estas dos variables. También es interesante observar que ante variaciones de carga el rango acotado de entrada de oxígeno es reducido lo que pudiera aliviar el estrés que pudiera sufrir el compresor del oxígeno como elemento final de control.

\section{Agradecimiento}

Este trabajo fue financiado por la Universidad Autónoma del Carmen UNACAR y por el Consejo Nacional de Ciencia y Tecnología CONACYT de México.

\section{Conclusiones}

El esquema propuesto demostró la capacidad para lidiar con los cambios de carga para un rango de operación. El flujo de oxígeno e mantuvo en valores adecuados, por lo que se redujo el riesgo de la falta de este. Además, la celda de combustible suministró energía de una manera mucho más uniforme y suave suficiente para el funcionamiento del motor.

Una de las ventajas de utilizar un Control por Modos Deslizantes sin retroalimentación de estados en el lado del sistema Celda-Convertidor es por un lado simplificar el número de sensores y por lo tanto el número de ecuaciones $\mathrm{y}$, por otro lado, llegar a un esquema muy simple de implementar con propiedades robustez lo que lo hace factible para futuras aplicaciones móviles comerciales. En este mismo sentido, la estrategia de control para el sistema Convertidor-Motor seguirá siendo el enfoque clásico PI debido a las mínimas variaciones de incertidumbre cuando se utilizan motor de reducida capacidad.

\section{Trabajo futuro}

Uno de los retos a mejorar en este trabajo, es la reducción del chatering sin incrementar el número de componentes de diseño que amerita incrementar la complejidad del control.

\section{Referencias}

Alnaib, A. M. I., Sultan, N. S., \& Mahmood, O. T. (2018). Design a fuel cell based drive dc motor for electric vehicle applications. International Journal of Engineering \& Technology, 7(4), 2081-2087.
Alnaib, A. M. I., Sultan, N. S., \& Mahmood, O. T. (2018). Design a fuel cell based drive dc motor for an electric vehicle application. International Journal of Engineering \& Technology, 7(4), 2081-2087.

Bordons, C., Arce, A., \& Del Real, A. J. (2006, June). Constrained predictive control strategies for PEM fuel cells. In 2006 American Control Conference (pp. 6-pp). IEEE.

Feroldi, D., Serra, M., \& Riera, J. (2006). Control de sistemas basados en pilas de combustible tipo PEM. Cataluña: Universidad Politécnica de Cataluña.

Garcia-Gabin, W., Dorado, F., \& Bordons, C. (2010). Real-time implementation of a sliding mode controller for air supply on a PEM fuel cell. Journal of process control, 20(3), 325-336.

Glazer, M. N., Oprean, I. M., \& Băţăuş, M. V. (2013). Modeling and Analysis of a Fuel Cell Hybrid Vehicle. In Proceedings of the FISITA 2012 World Automotive Congress (pp. 847858). Springer, Berlin, Heidelberg.

Hatti, M., \& Tioursi, M. (2009). Dynamic neural network controller model of PEM fuel cell system. International Journal of Hydrogen Energy, 34(11), 5015-5021.

Hirschenhofer, J. H., Stauffer, D. B., Engleman, R. R., \& Klett, M. G. (1998). Fuel cell handbook. Kunusch, C., Puleston, P., \& Mayosky, M. (2012). Sliding-Mode control of PEM fuel cells. Springer Science \& Business Media.

Larminie, J., Dicks, A., \& McDonald, M. S. (2003). Fuel cell systems explained. Chichester, UK: J. Wiley.

O'hayre, R., Cha, S. W., Prinz, F. B., \& Colella, W. (2016). Fuel cell fundamentals. John Wiley \& Sons.

Pukrushpan, J. T., Stefanopoulou, A. G., \& Peng, H. (2004). Control of fuel cell power systems: principles, modeling, analysis and feedback design. Springer Science \& Business Media.

Shtessel, Y., Edwards, C., Fridman, L., \& Levant, A. (2014). Sliding mode control and observation (pp. 163-166). New York: Springer New York.

CERVERA-CEBALlOS, María, RODRÍGUEZ-BLANCO, Marco A., VAZQUEZ-ÁVILA, José L. y ALAZKI, Hussain. Control por Modos Deslizantes de la Relación de Exceso de Oxígeno en una PEMFC Interconectada a un Motor de CD mediante un Convertidor Boost. Revista de Aplicaciones de la Ingeniería. 2019 
Simon, A. N. G., \& Oliva, A. (2005). Power switching converters. Marcel Dekker Limited.

Somkun, S., Sirisamphanwong, C., \& Sukchai, S. (2015). A DSP-based interleaved boost DCDC converter for fuel cell applications. International Journal of Hydrogen Energy, 40(19), 6391-6404.

Sonntag, R. E., Borgnakke, C., Van Wylen, G. J., \& Van Wyk, S. (1998). Fundamentals of thermodynamics (Vol. 6). New York: Wiley.

Tekin, M., Hissel, D., Pera, M. C., \& Kauffmann, J. M. (2007). Energy-management strategy for embedded fuel-cell systems using fuzzy logic. IEEE Transactions on Industrial Electronics, 54(1), 595-603.

Utkin, V. (1977). Variable structure systems with sliding modes. IEEE Transactions on Automatic control, 22(2), 212-222.

Williams, J. G., Liu, G., Chai, S., \& Rees, D. (2008). Intelligent control for improvements in PEM fuel cell flow performance. International Journal of Automation and Computing, 5(2), 145-151.

Young, K. D., Utkin, V. I., \& Ozguner, U. (1996, December). A control engineer's guide to sliding mode control. In Proceedings. 1996 IEEE International Workshop on Variable Structure Systems.-VSS'96- (pp. 1-14). IEEE.

Zhan, Y., Guo, Y., Zhu, J., \& Wang, H. (2008, October). Design and analysis of control system of a proton exchange membrane fuel cell directly connected with a DC motor. In Electrical Machines and Systems, 2008. ICEMS 2008. International Conference on (pp. 1706-1710). IEEE.

Zumoffen, D., \& Basualdo, M. (2010). Advanced control for fuel cells connected to a DC/DC converter and an electric motor. Computers \& Chemical Engineering, 34(5), 643-655. 